Journal of Patient-Centered

Volume 2

Issue 4 -- Integrative Medicine

Article 8

$11-20-2015$

\title{
Model Assessment and Development of Risk Stratification of Surgical Site Infection Following Cesarean Delivery for a High- Risk, Urban Population
}

Dakisha N. Lewis

Nicole P. Salvo

Kiley A. Bernhard

Danielle M. Greer

Follow this and additional works at: https://aah.org/jpcrr

Part of the Female Urogenital Diseases and Pregnancy Complications Commons, and the Obstetrics and Gynecology Commons

\section{Recommended Citation}

Lewis DN, Salvo NP, Bernhard KA, Greer DM. Model assessment and development of risk stratification of surgical site infection following cesarean delivery for a high-risk, urban population. J Patient Cent Res Rev. 2015;2:202. doi: 10.17294/2330-0698.1218

Published quarterly by Midwest-based health system Advocate Aurora Health and indexed in PubMed Central, the Journal of Patient-Centered Research and Reviews (JPCRR) is an open access, peer-reviewed medical journal focused on disseminating scholarly works devoted to improving patient-centered care practices, health outcomes, and the patient experience. 


\section{Proceedings of 2015 Aurora Scientific Day}

The following abstracts were presented at the 41st annual Aurora Scientific Day research symposium, held May 20, 2015, at Aurora Health Care Conference Center in Milwaukee, Wisconsin. Aurora Scientific Day provides a forum for original research conducted by residents, fellows, students, teaching and research faculty, and other allied health professionals at Aurora Health Care, a private nonprofit health care provider with 15 hospitals, 150 clinics and 70 pharmacies integrated throughout eastern Wisconsin and northern Illinois.

\section{FIRST PLACE ORAL PRESENTATION Model Assessment and Development of Risk Stratification of Surgical Site Infection Following Cesarean Delivery for a High-Risk, Urban Population}

Dakisha N. Lewis, Nicole P. Salvo, Kiley A. Bernhard, Danielle M. Greer

Department of Obstetrics and Gynecology, Aurora Sinai Medical Center and Aurora UW Medical Group; Center for Urban Population Health

Background: Surgical site infection (SSI) remains a major cause of morbidity despite efforts aimed at prevention and treatment. Risk stratification tools identify patients at greatest risk of SSI. Two models of stratification are: 1) the Centers for Disease Control and Prevention's National Healthcare Safety Network SSI Risk Index (NHSN), which assigns risk based on surgery duration, surgical wound contamination and physical status; and 2) the New Risk Stratification Schema (NRSS). The NRSS aimed to improve upon NHSN by incorporating five variables: diabetes control, body mass index (BMI), chorioamnionitis, methods of placental extraction and skin closure.

Purpose: Our objectives were to: 1) compare and evaluate NHSN and NRSS in terms of risk stratification outcomes in a high-risk urban population; and 2) develop a risk stratification model appropriate for assessing SSI risk in our population.

Methods: Data-related risk factors were gathered through chart review of all women who underwent cesarean delivery from September 2012 to October 2013. Using NHSN and NRSS models, we classified patients by risk of SSI following cesarean delivery. Logistic regression model effects represented 12 a priori risk factors in SSI, including BMI, diabetes, chorioamnionitis, delivery indication, use of chlorhexidine, preoperative antibiotics, timing of antibiotics, manual placental removal, antibiotic re-dosing, incision closure via staples, number of people present and surgery duration. Model-derived predicted values of SSI were used to stratify patients into low-, moderate- and high-risk categories. Strength of associations between SSI outcome and classified risks were examined. Agreement in risk classification between NHSN and NRSS, and each with our model, were assessed.

Results: Patients were normally distributed across the low- $(21.3 \%)$, moderate- $(55.9 \%)$ and high-risk $(22.9 \%)$ categories of NHSN, but under NRSS were restricted to moderate- $(33.6 \%)$ and high-risk (66.4\%) categories. While both methodologies produced results strongly associated with SSI $(\mathrm{P}<0.0001)$, agreement in SSI risk occurred for only $46.3 \%$ of patients. Modeling efforts established chorioamnionitis, BMI and surgery duration as the three most significant predictors of SSI.

Conclusion: While both NHSN and NRSS produced results strongly associated with SSI, distribution of patients was shifted toward high-risk in the NRSS arm. Our stratification model is a simplification of the NRSS, utilizing only three highly significant predictors: chorioamnionitis, BMI and surgery duration.

\section{SECOND PLACE ORAL PRESENTATION Operating Room First Start Efficiency Throughout a Large Urban Hospital System}

Callie Cox Bauer, Kiley A. Bernhard, Danielle M. Greer, Scott Kamelle

Department of Obstetrics and Gynecology, Aurora Sinai Medical Center; Center for Urban Population Health; Gynecologic Oncology, Aurora Health Care

Background: Operating room delays decrease health care system efficiency and increase hospital costs. Data on delays in a multihospital system are sparse.

Purpose: In an effort to improve our operating room efficiency, we investigated operating room delays, the causes and the impending financial impact.

Methods: A retrospective analysis on first case-of-the-day surgeries at three hospitals during 2013 was conducted. Delays were defined as in-room time being after scheduled surgery start time. Length of delay and causes were recorded. Patient demographics, body mass index, hospital facility, total number of procedures, provider specialty and time of patient arrival were incorporated into a logistic regression model to identify significant variables. Hosmer-Lemeshow was used to measure goodness-of-fit and predictive power. Cost was calculated using published estimates.

Results: 5,607 cases were examined and $88 \%$ were delayed. Surgeons (21\%), anesthesiologists $(6.17 \%)$, patients $(5.42 \%)$, staff $(3.60 \%)$, facility $(2.10 \%)$ and other $(2.35 \%)$ were identified as causes. Mean time for patient arrival to surgery was $104.57 \mathrm{~min}$. Mean time between arrival and 